\title{
A Novel Behavior Steganography Model Based on Secret Sharing
}

\author{
Hanlin Liu, National University of Defense Technology, Hefei, China \\ Jingju Liu, National University of Defense Technology, Hefei, China \\ Xuehu Yan, National University of Defense Technology, Hefei, China \\ Lintao Liu, National University of Defense Technology, Hefei, China \\ Wanmeng Ding, National University of Defense Technology, Hefei, China \\ Yue Jiang, National University of Defense Technology, Hefei, China
}

\begin{abstract}
This article proposes a novel behavior steganography model based on secret sharing, the main idea of which is to use secret messages as random elements in the secret sharing process to generate shadow images. Based on the introduced model and analyzing two secret image sharing algorithms threshold secret image sharing (SIS) and threshold visual secret sharing (VSS), two specific behavior steganography schemes are presented, which are implemented by utilizing secret sharing behavior. In the embedding phase, the random selection behavior is employed to hide secret messages. In the extraction phase, when the secret image is recovered from shadow images, secret messages can also be extracted successfully. The contribution of the authors model is that two secret information transmission channels are opened, which provides a large amount of hidden capacity and has loss tolerance and so on. Experimental results and analyses demonstrate the effectiveness of the proposed scheme. It has both good imperceptibility and large capacity, but the robustness of their scheme is poor.
\end{abstract}

\section{KEYWORDS}

(k, k) Threshold VSS, (k, n) Threshold SS, Behavior Steganography, Good Imperceptibility, Large Capacity, Secret Sharing

\section{INTRODUCTION}

Information hiding is an art of hiding secret messages in another seemingly innocuous carrier (Johnson, Duric \& Jajodia, 2012), which is a powerful technique that can enhance security in data transferring and archiving. Throughout history, a multitude of methods have been used to hide information. With the development of the Internet and other new technologies, digital steganography technique, which is used to embed the secret message into digital multimedia, is gradually rising. It has developed a strong basis for the area of steganography with a growing number of applications for digital fields like digital rights management, covert communications, annotation etc. So far, various researches on steganography have been carried out on storage media, such as text, image, audio, and video.

Compared with security technologies, such as encryption and secret sharing, the aim of information hiding conceals the existence of secret messages to ensure that the secret information transmission unbeknown. Since when an attacker doesn't know the existence of secret messages, he won't generate the idea of attacking it. Moreover, the information hiding method must also ensure 
the successful extraction of the secret information. Thus, there are three metrics that evaluate a steganography scheme (Divya \& Reddy):

1. Imperceptibility evaluates how well a secret message is embedded into the carrier. The difference between carrier after hiding and before hiding should remain negligible.

2. Robustness indicates the ability of secret messages to resist against attacks.

3. Capacity means the amount of secret information that can be embedded into the original carrier without affecting the perceptual quality of carrier.

The traditional steganography is to hide the secret message embedded in the digital multimedia, and in recent years, coverless steganography and behavior steganography gradually rise. So, this paper intends to propose a novel behavior steganography model based on the random selection behavior of secret sharing.

In many SIS schemes, shadow images need to be generated according to some random numbers whose values have little effect on the sharing and recovery of secret images and how to choose a random number is controllable. So, the selection of random numbers, this behavior, can be considered as a way of steganography. On the basis of this idea, this paper presents a behavior steganography model based on secret image sharing. In the embedding phase, the random selection behavior is employed to hide the secret message. In the extraction phase, when the secret image is recovered from shadow images, the secret messages can also be extracted successfully. Experimental results and analyses demonstrate the effectiveness of the proposed model. In comparison with traditional steganography methods, our contributions are summarized as follows:

1. First, we explore the behavior steganography model based on secret sharing. This model combines information hiding and secret sharing, and secret messages are hidden in parallel with secret image sharing, which means that in a communication process, two covert channels are opened;

2. There are lots of random elements participating in the secret sharing process. And we embed secret messages in random elements, which can provide a large amount of hidden capacity;

3. The steganography process does not require the original carrier, and secret messages are just embedded during the secret sharing process. So, the risk of being analyzed reversibly is reduced because of no comparison with original carrier. In addition, the shadow image obtained by this method is basically the same as that obtained by the original method;

4. Traditional steganography does not have loss tolerance. Our scheme based on secret sharing makes up the deficiency of steganography. Even if part of the shadow images is lost, secret messages can still be successfully extracted;

5. This model is applicable to a variety of secret image sharing algorithms. The two schemes proposed in this paper are just examples of the combination of our model and specific secret sharing algorithms, and more secret sharing algorithms can be applied to this model.

The proposed behavior steganography model is very similar to multi-secret sharing schemes as mentioned above, both of which can achieve more secrets sharing or hiding in just a single secret sharing scheme. However, we introduce a novel steganography model to achieve multi-secret sharing based on the random selection behavior of secret sharing. The behavior steganography technique is the basis of our proposed scheme, which is firstly proposed in the area of secret sharing.

The relation between secret sharing and steganography is discussed academically. Our work utilizes the sharing behavior to achieve information hiding. We do not focus on the relation between secret sharing and steganography.

The remainder of this paper is organized as follows: Section II discusses some existing methods, and Section III introduces some preliminaries. The proposed model and concrete schemes are in Section IV. In Section V experimental results of proposed scheme are discussed and analyzed. Finally, Section VI concludes this paper. 


\section{THE RELATED WORK}

Steganography techniques utilize the $20^{\text {th }}$ century's inventions - computers and networking, and four main trends of the so-called digital information hiding can be distinguished: digital media information hiding; linguistic information hiding; file system information hiding; and network information hiding (Zieliska, Mazurczyk \& Szczypiorski, 2014). However, the information hiding methods above are to embed the secret messages by modifying the existing carrier and the differences between the stego-carrier and the original carrier make its safety declining and may become a breakthrough of steganalysis. In (Zhang, Qian \& Li, 2016), Zhang et al. proposed a novel information hiding method-structured information hiding including semi-structured and completely structured, which refers to the fact that the stego-carrier can be directly generated by secret information in accordance with certain rules without pre-specifying the original carrier, and the stego-carrier may not represent the real objective things, but compared with the normal content it should be indistinguishable. Zhang also (Zhang,2017) proposed a behavior steganography in social network. In the method, a sender makes "love" marks on the news published by his friend with given rates for representing the secret data, and a receiver who is a friend of the sender can extract the secret data from a part of the sender's "love" marks. Nevertheless, it is unfit for a large amount of secret data. In this paper, we propose a behavior steganography scheme based on secret sharing, in which the stego-carriers are driven by secret information.

As two important ways of secret information transmission, predecessors have taken into account the combination of information hiding and secret sharing to transmit secret information. In ( $\mathrm{Li}$, El-Latif, Yan, Wang \&Niu, 2012), a new lossless secret sharing method based on steganography was proposed. The new method generates shares from the cover image and secret image, and then embeds the shares into cover images obtaining stego-shadow images based on 24-ary notational system. Similarly, in (Jagadeesh, Nandakumar, Harmya \& Anju, 2011), Jagadeesh et al. proposed a novel approach to secret image sharing based on a (t, n) threshold scheme with steganography, and the steganography method is utilized to embed the shadows indifferent cover images. Additionally, Kongetal. (Kong, Zhang, Meng, Zheng \& Lu, 2007) presented a scalable secure scheme for sharing and hiding secret image, in which the shadows are concealed in R, G and B channels of each cover image. The three methods above have a common feature: secret sharing and information hiding are used continuously and then the generated shadows are embedded in the carrier images. There is no real combination of the two technologies.

Furthermore, some methods in transfer domain are also proposed. In (Banik \& Bandyopadhyay, 2016), Baniketal. proposed a new technique of image steganography, which is using Lorenz Chaotic Encryption to encrypt the secret message, 3 level discrete wavelet transform to hide encrypted data and visual cryptography to share stego-image in secret communication. In (Khosravi \& Ghandali, 2011), Khosravi et al. proposed a method in which a secret image is shared into some shadow images. Then, shadow images and Fletcher-16 check sum of shares are hidden into cover images using an integer wavelet based on steganography technique. In (Cao, 2011), a new binary image hiding scheme was proposed by $\mathrm{Cao}$ and Askar, which hides a binary image into several cover images. Based on the characteristics of the binary image whose value is 1 or 0 , this scheme combines multi-secret sharing with run length encoding (RLE) and DCT. According to the biggest size of cover images, this scheme divides RLE length of the shadow image into $n$ blocks, and then embeds the $n$ blocks of secret information in cover images under the control of $n$ keys. By contrast, these methods in transform domain are stable against serious attacks or have other better properties. But they did not achieve the true integration of the two technologies, either.

In summary, most of the existing methods are used to hide shares through information hiding, and information hiding is just a way of shadow images transmission. And there are few researches on information hiding utilizing secret sharing process. Li et al (Li, Ma, Su \& Liu, 2012) proposed a multi-threshold image secret sharing scheme which is intended to consider hiding messages in SIS and 
is similar to our model, but its scope of application is limited to visual cryptography scheme(VCS). In order to overcome the above problems, we propose a behavior steganography unified model based on secret image sharing, and then present two behavior steganography schemes using specific secret sharing algorithms. Our steganography scheme is not only applicable to more secret sharing methods, but also has considerable steganographyic capacity.

\section{PRELIMINARIES}

In this section, we exhibit some preliminaries for our work. As an important technology of information security, secret image sharing encrypts the images into different noise-like shadow images and distributes them to participants. Since the shadow images are noise-like, and every single shadow image gives no clue about the secret, hence, the secret is well protected by secret sharing. Visual cryptographic scheme (VCS) (Wang, Arce \& DiCrescenzo, 2009; Naor \& Shamir, 1995; Yan, Wang $\&$ Niu, 2014) and Shamir's polynomial-based scheme (Shamir, 1979; Thien \& Lin, 2002; Lin \& Lin, 2007; Yang \& Ciou, 2010; Li, Yang, Wu, Kong \& Ma, 2013), are the primary branches in secret image sharing. In our paper, the proposed model can be applied to different secret sharing algorithms, and we just select two representative algorithms as examples. And in order to facilitate readers to understand the article, some notations are explained as shown in Table 1.

In a $(k, n)$ threshold SIS, the secret image $S$ with size of $W \times H$ is encrypted into total $n$ shares $S C_{1}, S C_{2}, \cdots S C_{n}$, while the revealed secret image $S^{\prime}$ is revealed from $t\left(k \leq t \leq n, t \in \mathrm{Z}^{+}\right)$shares. $S(i, j) \in[0, P-1]$, where $[0, P-1]$ means the pixel value range and $P$ denotes the maximum pixel value, such as, for VSS $P=2$ and $P=251,256$ or a suitable prime number for gray image or color image sharing.

In the following, $\otimes$ and $\oplus$ indicate Boolean OR and XOR operations, respectively.

\subsection{Polynomial-Based $(k, n)$ Threshold SIS}

We assume the now processing gray secret image pixel value is $s$, and then to encode $s$ into $n$ pixels assigned to $n$ corresponding shares by Shamir's first polynomial-based SIS scheme. The following Steps are repeated until processing all the secret pixels.

Table 1. Notation description

\begin{tabular}{|l|l|}
\hline Notation & Meaning of notation \\
\hline$(k, n)$ & $n$ is the number of shadow images and $k$ is the threshold \\
\hline$(k, k)$ & the number of shadow images and the threshold both are $k$ \\
\hline$W, H$ & the size of secret image \\
\hline$M$ & The secret image \\
\hline$S C_{t}$ & the secret message \\
\hline$S^{\prime}$ & the $t$ th shadow image \\
\hline$S(i, j)$ & the revealed secret image $S$ \\
\hline
\end{tabular}


Step 1:Forthe now processing pixel value $s=S(i, j)$, in order to split $s$ into shared pixels $s c_{i}$, we construct a $k-1$ degree polynomial

$g(x)=\left(a_{0}+a_{1} x+\cdots+a_{k-1} x^{k-1}\right) \bmod P$

where $a_{0}=s, a_{i}$ is random for $i=1,2, \cdots k-1$ and $P=251$.

Set

$s c_{1}=g(1), \cdots, s c_{i}=g(i), \cdots, s c_{n}=g(n)$

In the recovery of polynomial-based SIS, for any given $k$ pairs among the $n$ pairs $\left\{\left(i, s c_{i}\right)\right\}_{i=1}^{n}$, where

3.2. $i$ may be served as an order label for the $i$ th owner, we can obtain the coefficients of $g(x)$ based on Lagrange's interpolation, and then get $s=g(0)$. The processing repeats until all pixels of the secret image are processed. And the secret image $S$ cannot be over all revealed with less than $k$ shares. $(k, k)$ ThresholdVSS

In random grids visual secret sharing (RGVSS) (Kafri\&Keren,1987),1 indicates black pixel and 0 denotes white pixel. The generation Steps and revealing phase of one popular original $(2,2)$ RGVSS are described as follows.

Step 1: Construct $1 \mathrm{RG} S C_{1}$ randomly.

Step 2: Compute $S C_{2}$ as in Equation (3), where $\overline{S C_{1}(i, j)}$ denotes bit-wise complementary operation of a pixel $S C_{1}(i, j)$.

Revealing phase: $S^{\prime}=S C_{1} \otimes S C_{2}$ from Equation (4).If the secret pixel $s=S(i, j)$ is 1 , the recovery bit $s c_{1} \otimes s c_{2}=1$ is black. If the certain secret pixel is 0 , the recovery bit $s c_{1} \otimes s c_{2}=S C_{1}(i, j) \otimes S C_{1}(i, j)$ has half chance to be black or white because $s c_{1}$ is generated randomly.

$$
\begin{aligned}
& S C_{2}(i, j)= \begin{cases}S C_{1}(i, j) & \text { if } S(i, j)=0 \\
S C_{1}(i, j) & \text { if } S(i, j)=1\end{cases} \\
& S^{\prime}(i, j)=S C_{1}(i, j) \otimes S C_{2}(i, j)= \begin{cases}S C_{1}(i, j) \otimes S C_{1}(i, j) & \text { if } S(i, j)=0 \\
S C_{1}(i, j) \otimes \overline{S C_{1}(i, j)}=1 & \text { if } S(i, j)=1\end{cases}
\end{aligned}
$$

We remark that, Equation (3) is equal to $s c_{2}=s c_{1} \oplus s$ and Due to if $s=s c_{1} \oplus s c_{2} . s=0 \Rightarrow s c_{2}=s c_{1} \oplus 0 \Rightarrow s c_{2}=s c_{1}$, andif $s=1 \Rightarrow s c_{2}=s c_{1} \oplus 1 \Rightarrow s c_{2}=s c_{1}$. Thus, the same equation can be extended to $s=s c_{1} \oplus s c_{2} \oplus \cdots \oplus s c_{k}$ so that $(k, k)$ threshold RGVSS is achieved. 


\section{A COMMON BEHAVIOR STEGANOGRAPHY MODEL BASED ON SECRET IMAGE SHARING}

Shadow images are generated according to some random numbers whose values have little effect on the sharing and recovery of secret image. So, the selection of random numbers, this behavior, can be considered as a way of steganography. Hereon, we will introduce a common behavior steganography model based on secret image sharing and then analysis its performance.

\subsection{The Proposed Model}

The introduced common behavior steganography model is shown in Figure.1. And the model can be divided into two parts, the embedding process and the extraction process.

In the embedding process, the shadow images are generated, at the same time, the secret message is embedded into those random elements. The concrete steps of the embedding process are as follows:

1. First, the secret messages are binarized by encryption, after which the binary secret messages are segmented into random elements according to SIS behavior. At the same time, each image pixel value is obtained from the secret image;

2. Second, $n$ shadow pixels are generated using the secret sharing algorithm with the random elements. It should be noted that the length of the random element is determined by the secret sharing algorithm;

3. Finally after all the image pixels have been processed, we get $n$ shadow images which will be distributed to $n$ participants through different channels.

In the extraction process, the secret image can be restored and the secret message can be extracted successfully. The concrete steps of the extraction process are as follows:

1. When the number of shadow images reaches the threshold, each shadow image pixel is manipulated by SIS algorithm;

2. While recovering the secret image, we can also extract the random elements;

3. After decimalization and decryption, the random elements are converted into secret messages.

We can see that there are two elements including secret messages and secret image involved in the embedding process, and both secret messages and secret image are extracted in the extraction process, which means that two information transmission channels are opened in a single act. Steganography and dual channel communication are achieved by using random elements in secret sharing, which is also a great significance of our model.

Qualitative performance analysis: In section I, we have introduced three criteria of information hiding: imperceptibility, robustness and capacity. Thus, we will qualitatively analyze the performance of the steganography model from these three aspects.

1. In this model, since the carrier is not already present, which is generated by the secret messages. Therefore, shadow images do not contain any traces of secret messages, and it is also very difficult to steganalysis.

2. The correct extraction of the secret messages is determined by the shadow image. If the shadow image is attacked and the pixel value of the shadow image is modified, the extraction of the secret message may be affected. Therefore, the robustness of the model is not satisfactory.

3. In the phase of secret image sharing, such as Shamir's first polynomial-based SIS scheme, shadow pixel values and $a_{1}, a_{2}, \cdots a_{k-1}$ used in the process of generating shadow images are uncertain. These uncertain values can be determined by the behavior of random selection. So, the capacity 
is determined by the number of uncertain values, and in this process a lot of random values are used to generate shadow images, thus the steganographic capacity is still considerable.

\subsection{A Concrete Behavior Steganography Scheme Based on $(k, n)$ Threshold SIS}

In this subsection, we will concretize the SIS algorithm of the model, and use the $(k, n)$ threshold SIS to replace it. So, a random behavior steganography scheme based on $(k, n)$ threshold SIS is presented, the embedding phase of which is described in Algorithm 1. And Algorithm 1 is corresponding to the embedding process of Figure 1.

Algorithm 1. A behavior steganography scheme based on $(k, n)$ threshold SIS in embedding phase

Input: (1) a $\mathrm{W} \times \mathrm{H}$ secret image $S$ which will be shared, (2) secret messages $M$ which will be embedded, (3) threshold parameters $(k, n)$, (4) encryption key.

Output: $n$ stego-shadow images $S C_{1}, S C_{2}, \cdots S C_{n}$.

Step 1: Encrypt the secret messages $M$ and binarize them, then divide the binary secret messages $M^{\prime}$ into segments for every 7 or 8 bits. And convert each segment into decimal data $m_{1}, m_{2}, \cdots m_{l}, l=$ length $\left(M^{\prime}\right) / 7$ or $l=$ length $\left(M^{\prime}\right) / 8$

Step 2: For each position $S(w, h),(w, h) \in\{(w, h) \mid 1 \leq w \leq W, 1 \leq h \leq H\}$, repeat Step 3 .

For the current secret pixel $s=S(w, h)$, use $(k, n)$ threshold SIS to split $s$ into $k$ shared pixels, which is generated by the $k-1$ degree polynomial

$g(x)=\left(a_{0}+a_{1} x+\cdots+a_{k-1} x^{k-1}\right) \bmod P$

where $a_{0}=s, a_{i}$ equals to current unprocessed segment. And if we finish embedding $M^{\prime}, a_{i}$ is assigned to a random integer between 1 and 128 or 251. $S C_{t}(w, h)=g(z), z \in\{z \mid 1 \leq z \leq n\}$, $t \in\{z \mid 1 \leq t \leq n\}$.

Step 4: Generate $n$ shadow images and distribute them to different participants via different channels.

Figure 1. The proposed behavior steganography model

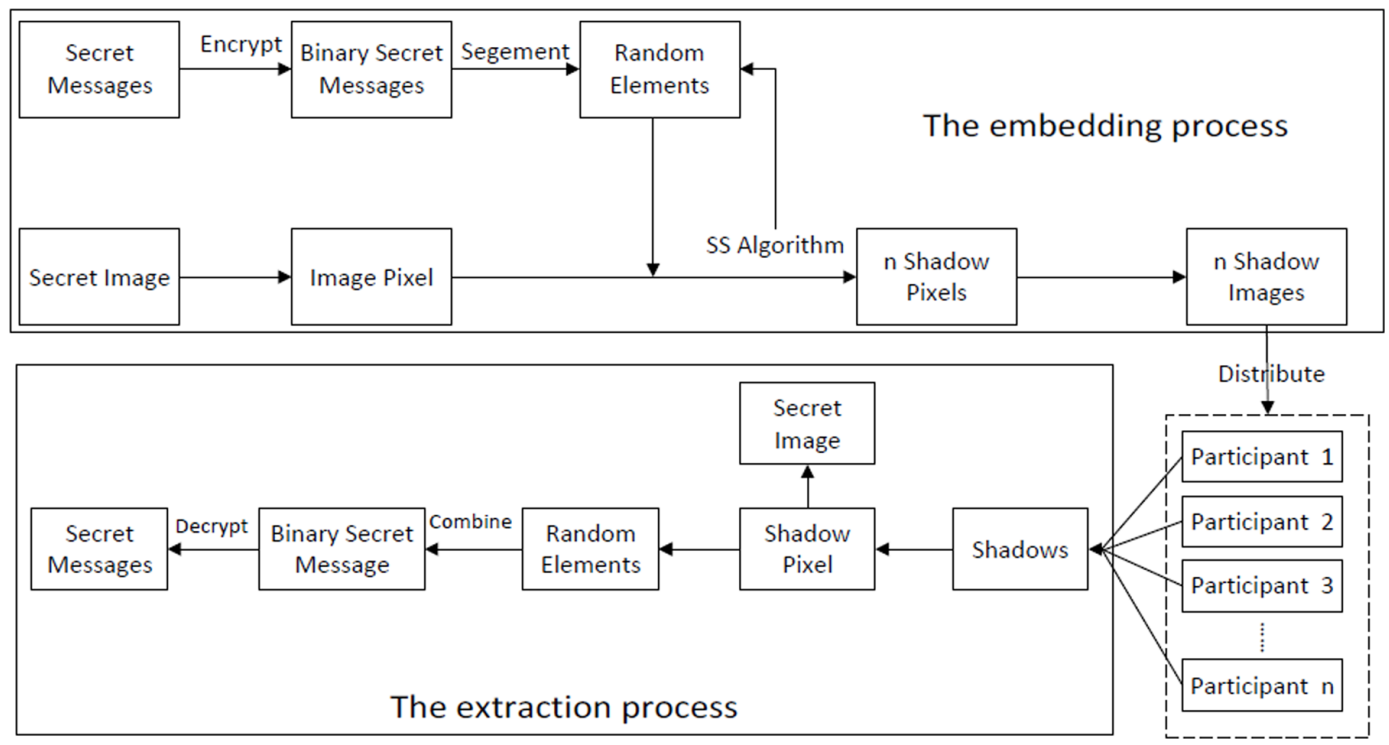


In Algorithm 1, we remark that.

1. In this algorithm, the polynomial coefficients $a_{2}, a_{3}, \cdots a_{k-1}$ can be assigned to random values. So, the polynomial coefficients are determined by selecting random value sand secret messages are embedded in this process.

2. In step 1, if the secret messages are text, each segment consists of seven bits and if an image, it consists of eight bits.

3. Here, length $(\cdot)$ is used to calculate the length of the binary secret messages.

4. In step3, when $i>W \times H$, if the secret messages are text, $a_{i}$ is a random integer between 1 and 128 , and if an image, $a_{i}$ is a random integer in the range of 1 and 251.

The extraction phase of this scheme can be described in Algorithm 2, which is corresponding to the extraction process of Figure 1.

Algorithm 2. A random behavior steganography scheme based on $(k, n)$ threshold SIS in extraction phase

Input: (1) $k$ or more shadow images which are randomly selected from $n$ secret shadow images $S C_{1}, S C_{2}, \cdots, S C_{n}$ (2) decryption key.

Output: The secret messages $M$ and the original secret image $S$.

Step 1: Select $k$ or more than $k$ shadows, and note down their labels $x_{1}, x_{2}, \cdots, x_{k}$.

Step 2: For each position $(w, h) \in\{(w, h) \mid 1 \leq w \leq W, 1 \leq h \leq H\}$, repeat Step $3-5$.

Step3: According to the $k$ or more labels, construct the $k$ or more corresponding polynomials based on the Lagrange's interpolation.

Step 4: Get the coefficient $a_{0}$ by solving polynomials, and set the pixel $S(w, h)$ as the value $a_{0}$.

Step5: Get the remaining coefficients $a_{i}$ and set them as the value of encrypted secret messages $M^{\prime}$.

Step 6: Decrypt $M^{\prime}$, and get the original secret messages $M$.

Step 7: Output the original secret messages $M$ and the secret image $S$.

It is worth noting that the number of shadow images participating in extraction phase cannot be less than $k$, if not, both the secret image $S$ and secret messages $M^{\prime}$ are not available.

\subsection{A Concrete Behavior Steganography Scheme Based on $(k, k)$ Threshold VSS}

In this subsection, we implement the SIS algorithm of the model, and use the $(k, k)$ threshold VSS to replace it. So, a behavior steganography scheme based on $(k, k)$ threshold VSS is presented, and the embedding phase of which is described in Algorithm 3. And Algorithm 3 is corresponding to the embedding process of Figure 1.

In Algorithm 3, it should be noted that: in step 2, length $(\cdot)$ is used to calculate the length of the binary secret messages.

Algorithm3. A random behavior steganography scheme based on $(k, k)$ threshold VSS in embedding phase

Input: (1)a W $\times \mathrm{H}$ binary secret image $S$ which will be shared, (2) secret messages $M$ which will be hidden, (3) threshold parameters $(k, k)$.

Output: $n$ shadow images $S C_{1}, S C_{2}, \cdots S C_{n}$.

Step 1: Encrypt the secret messages $M$ and binarize them to get the binary secret messages $M^{\prime}$

Step 2: For each position $S(w, h),(w, h) \in\{(w, h) \mid 1 \leq w \leq W, 1 \leq h \leq H\}$, repeat Step 3 - 4 .

Step3: $S C_{k}(w, h)$ is randomly assigned to 0 or 1 . 
$S C_{v}(w, h)$ equals to current unprocessed binary secret bit for $v \in\{v \mid 1 \leq v \leq k-1\}$. And if we finish embedding the binary secret messages $M^{\prime}, S C_{v}(w, h)$ is randomly assigned to 0 or 1 for $v \in\{v \mid 1 \leq v \leq k-1\}$.

Step 4: If $S C_{1}(w, h) \oplus S C_{2}(w, h) \oplus \cdots \oplus S C_{k}(w, h) \neq S(w, h)$, then flip $S C_{k}(w, h)$.

Step5: Generate $k$ shadow images and distribute them to different participants via different channels. ( $\mathrm{k}, \mathrm{n}$ ) threshold VSS is possible in our model, the embedded capacity is dependent on the number of random bits.

Algorithm 4. A random behavior steganography scheme based on $(k, k)$ threshold VSS in extraction phase

Input: The $k$ shadow images $S C_{1}, S C_{2}, \cdots, S C_{k}$

Output: The secret messages $M$ and the original secret image $S$

Step 1: Select $k$ shadows which is used to recover secret image and extract secret messages.

Step 2: For each position $(w, h) \in\{(w, h) \mid 1 \leq w \leq W, 1 \leq h \leq H\}$, repeat Step 3 .

Step 3: Set $S(w, h)=S C_{1}(w, h) \oplus S C_{2}(w, h) \oplus \cdots \oplus S C_{k}(w, h)$.

Step 4: Combine $S C_{1}, S C_{2}, \cdots, S C_{k-1}$ to obtain $M^{\prime}$. $M^{\prime}$ is decrypted and converted to get secret messages $M$.

Step 5: Output the original secret messages $M$ and the secret image $S$.

The extraction phase of this scheme can be described in Algorithm 4, which is corresponding to the extraction process of Figure 1.

It is important to emphasize that the receiver must obtain all the shadow images, otherwise the secret image cannot be recovered.

The above two schemes are only the specific application of the proposed model for different secret sharing algorithms. And in the above two scheme, the former is based on Shamir's polynomialbased $(k, n)$ threshold SIS, which applies to grayscale images and has a larger capacity. The latter is based on $(k, k)$ threshold VSS, which is applied to binary image. Although the capacity of the second scheme is smaller than that of the first scheme, the computational complexity of the second scheme is low when recovering the secret image and extracting the secret information.

\subsubsection{Quantitative Performance Analysis}

In order to evaluate the proposed schemes more objectively, we use $E_{p}$, BER and PSNR to make a quantitative performance analysis.

$E_{p}$ is measure by bit per pixel $(B P P)$, represents the number of bits that can be embedded average per pixel, which is calculated by Equation (5).

$$
E_{p}=\frac{L}{P \times \mathrm{n}}
$$

Where $L$ is the length of binary secret messages. $P$ is the number of pixels in the secret image, which is equal to $W \times H$, and $n$ is the number of shadow images.

BER refers to the bit error rate, which is the ratio of the number of error bits in the transmission process to the total number of bits and used to evaluate the robustness of the algorithm. The formula is as Equation (6). 


$$
B E R=\left(\frac{l}{L}\right) \times 100 \%
$$

Where $l$ is the number of error bits.

If the secret message is an image, Peak Signal to Noise Ratio (PSNR) is also used to evaluate the robustness, which is calculated using Equation (7).

$$
P S N R=10 \log _{10}\left(\frac{255^{2}}{M S E}\right)
$$

Where MSE is the Mean Square Error, and it referred to the difference value between original secret messages and extracted messages, which is given in Equation (8).

$$
M S E=\frac{1}{W_{1} \times H_{1}} \sum_{i=1}^{W_{1}} \sum_{j=1}^{H_{1}}\left[P(i, j)-P_{a}(i, j)\right]^{2}
$$

Where $P(i, j)$ and $P_{a}(\mathrm{i}, \mathrm{j})$ are pixel values of the original secret messages and extracted secret messages. $W_{1}$ and $H_{1}$ are the width and height of the original secret messages (the extracted secret messages have the same size).

1. Imperceptibility(1): For the proposed scheme based on $(k, n)$ threshold SIS, since the encrypted secret message is almost a noise image, and if we take them as $a_{i}$ to participate in secret sharing, the quality of shadow images will not be affected, which can be verified by observing the pixel value distribution map of shadow images. And in the case of no attack, as long as the number of shadow image reaches the threshold, the secret messages are successfully extracted. (2) As for the proposed scheme based on $(k, k)$ threshold VSS, the encrypted binary secret messages are embedded in the first $k$ shadow images, which is also a noise image, and the values of the other binary shadow images are randomly generated. Therefore, theoretically, these shadow images are also noise images, so there is no secret image leakage.

2. Robustness (1) In the proposed scheme based on $(k, n)$ threshold SIS, whether $a_{1}, a_{2}, \cdots, a_{k-1}$ can be extracted correctly determines the accuracy of the secret messages, and the correctness of $a_{1}, a_{2}, a_{k-1}$ depends on the shadow image. Therefore, once the shadow images are attacked, it will directly lead to secret messages extraction failure. At the same time, the recovery of the secret images will also be negatively affected. (2) In the proposed scheme based on $(k, k)$ threshold VSS, because the secret message is embedded in the first $k$ shadow images, the attacks on shadow images will directly affect secret messages, as well as the recovery of the secret image. Therefore, for these two methods, their robustness may not be very good.

3. Capacity (1) It is mentioned that the capacity is determined by the number of uncertain values $a_{i}$. In the proposed scheme based on $(k, n)$ threshold SIS, sharing a secret image pixel requires $k-1$ uncertain values $a_{1}, a_{2}, \cdots a_{k-1}$. So, the maximum capacity is equal to $8 \times(k-1) \times W \times H$. That is, when the size of the secret image is constant, the greater the threshold, The greater the capacity. (2) As for the proposed scheme based on $(k, k)$ threshold VSS, capacity is equal to $k$ times the size of shadow image. If more shadow images instead of only the first shadow image are used to hide the secret message, capacity will multiply. 


\section{EXPERIMENTAL RESULTS AND ANALYSES}

In order to test and verify the effectiveness and performance of the proposed two schemes, we design the following experiments from three aspects: imperceptibility, robustness and capacity.

\subsection{Experiment of The First Scheme}

In this experiment, both the secret image and the secret message are with the size of $256 \times 256$, and we set $(k, n)$ to $(2,4)$, that is, the secret image and the secret message can be recovered as long as any two or more of the four shadow images are obtained.

\subsubsection{Imperceptibility Test}

In the condition of no attack, the experimental results are shown in Figure.2. The secret image and secret message are given in Figures.2 (a) and (b), and Figure.2 (c) is the encrypted secret message. Figures.2(d) - (g) display the 4 shadow images $S C_{1}, S C_{2}, S C_{3}$ and $S C_{4}$, which are noisy. Figures.2 (h) and (i) are the revealed secret image and extracted secret message when collecting $S C_{1}$ and $S C_{2}$ based on $(k, n)$ threshold SIS recovery. Image (c) is the result of image (b) encryption. The image (a) is shared into four shadow images (d) (g) using the pixel values in the image (c) as random elements, which is the most important step to realize the transmission of secret image and secret message. When the secret image (h) is recovered by the shadow images (d) and (e), the random element scan be calculated. Therefore, secret message (i) embedded in secret sharing process is extracted.

Experimental results show that the naked eye cannot see the meaning of the shadow images, which are indeed four noise images. The result of this experiment is because the encrypted secret message is random. When the secret message is used as $a_{1}, a_{2}, \cdots, a_{k-1}$ to generate shadow images, there is no correlation among shadow image pixels, so the shadow images are safe enough hand the scheme has good imperceptibility. And the results can also be displayed through the histogram Figure 3. The histogram of secret image and secret message are shown in Figures 3 (a) and (b), respectively. And Figures 3 (c) - (f) are the histogram of four shadow images $S C_{1}, S C_{2}, S C_{3}$ and $S C_{4}$.

The histogram reflects the distribution of pixel values. The smoother the histogram is, the more uniform the pixel value distribution is, and the closer the image is to the random noise. When the shadow images are close to random noise, it is shown that the shadow images do not leak the content of the secret image and the secret message, which proves the scheme has good imperceptibility.

From Figure 3, we can see that the histogram of four shadow images are random, all of which are not similar with Figures 3 (a) and (b) at all. Therefore, it can be concluded that the shadow images obtained by this scheme does not reveal the contents of the secret image and secret message, and the scheme has good imperceptibility.

\subsubsection{Robustness test}

Robustness is an important criterion to evaluate the performance of algorithm. In the experiment, common image processing are additive Gaussian noise with mean value of 0 and variance of 0.02 , additive salt and pepper noise with noise density of 0.04. And the test results are shown in the Figure 4 and Table 2.

Adding Gaussian noise with mean value of 0 and variance of 0.02 to the four shadow images, and then using the two shadow images to restore the secret image and extract the secret message, we will get the results as shown in Figures 4 (a)and(b). Figures 4 (a) and (b) are the revealed secret image and extracted secret message after additive Gaussian noise. And adding salt and pepper noise with noise density of 0.04 to the four shadow images, and then using the two shadow images to restore the secret image and extract the secret message, we will get the results as shown in Figures.4 (c) and (d). Figures 4 (c) and (d) are the revealed secret image and extracted secret message after additive 
Figure 2. Experimental results of the proposed scheme based on $(k, n)$ threshold SIS, where $k=2, n=4$ (a) The secret image; (b) The secret message; (c) The encrypted secret message; (d) - (g) four shadow images $S C_{1}, S C_{2}, S C_{3}, S C_{4}$; (h) Revealing result by $S C_{1}$ and $S C_{2}$; (i) Extracting result by $S C_{1}, S C_{2}$

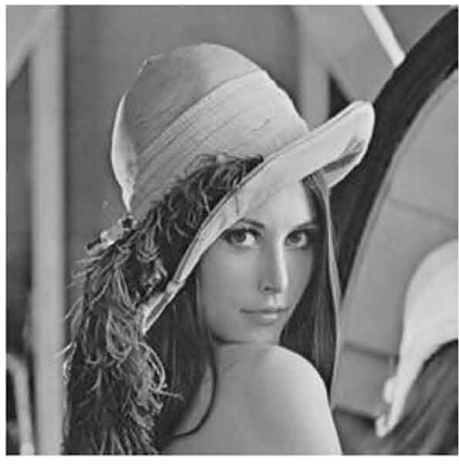

(a)

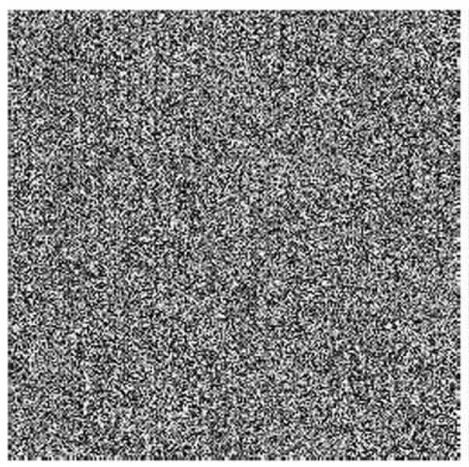

(d)

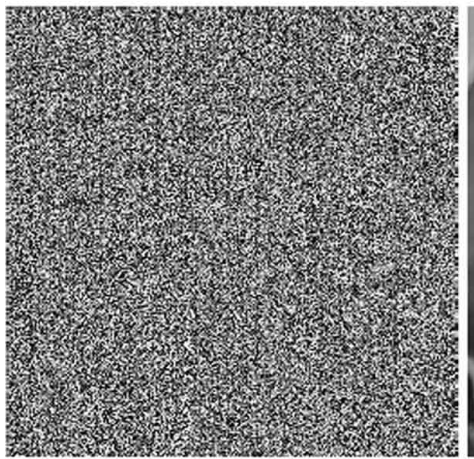

(g)

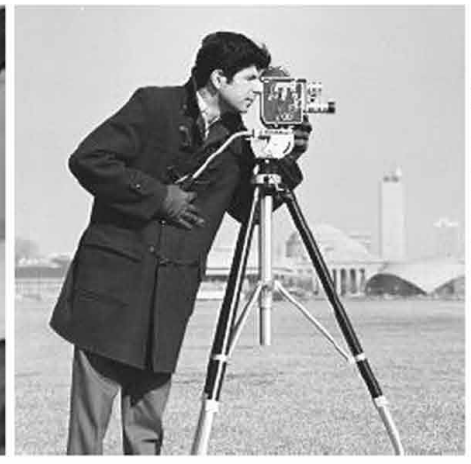

(b)

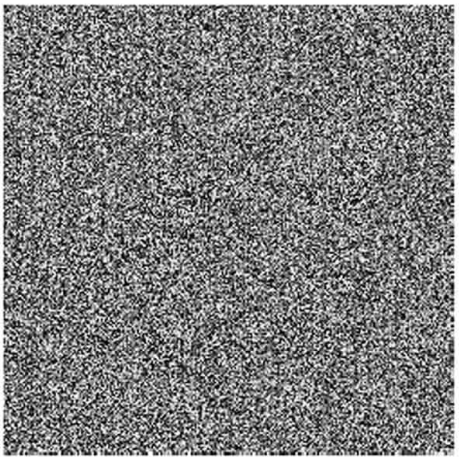

(e)

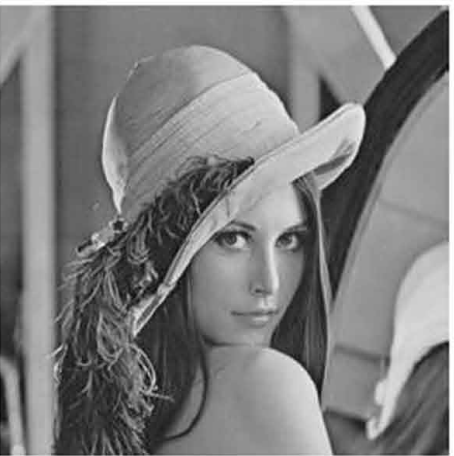

(h)

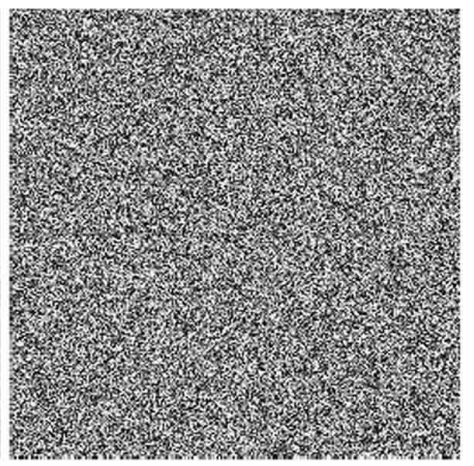

(c)

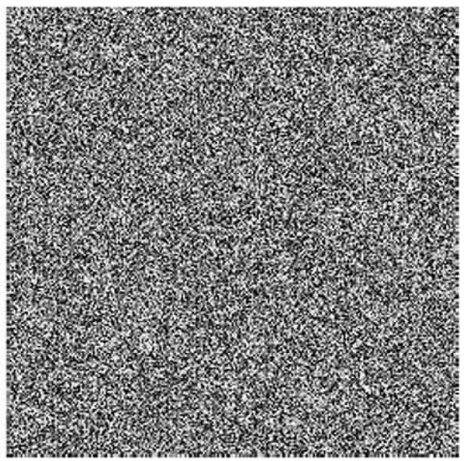

(f)

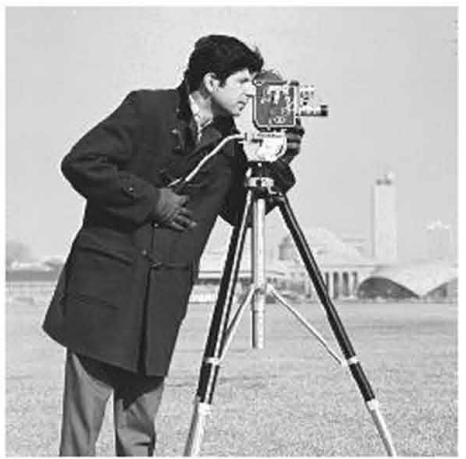

(i)

salt and pepper noise, respectively. It can be seen from Figure 4 and Table 2 that the ability of the scheme to resist the additive Gaussian noise and additive salt and pepper noise attack is obviously very poor. Since the secret message are extracted by the Lagrange interpolation method, and when the noise changes the pixel values of the shadow images, the secret message extracted according 
Figure 3. (a) The histogram of secret image; (b) The histogram of secret message; (c) - (f) The histogram of four shadow images $S C_{1}, S C_{2}, S C_{3}, S C_{4}$

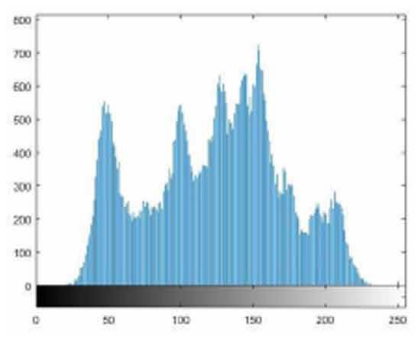

(a)

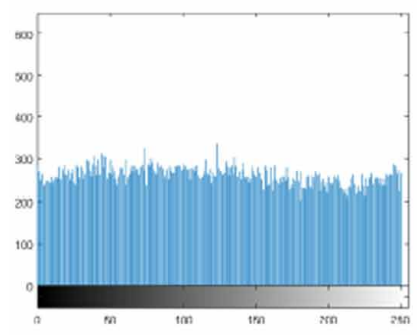

(d)

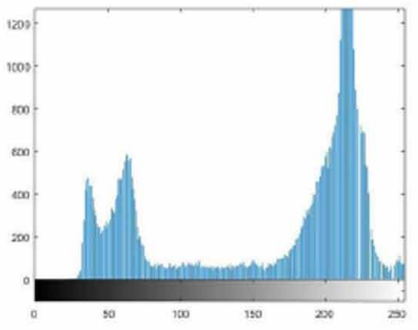

(b)

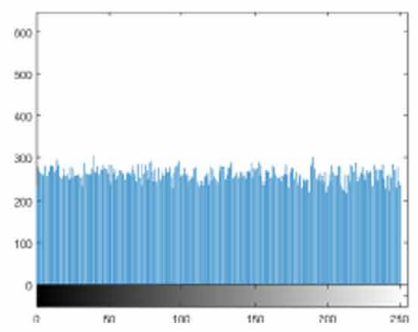

(e)

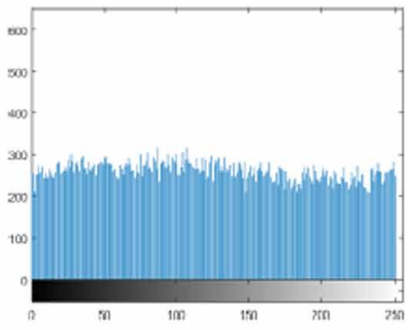

(c)

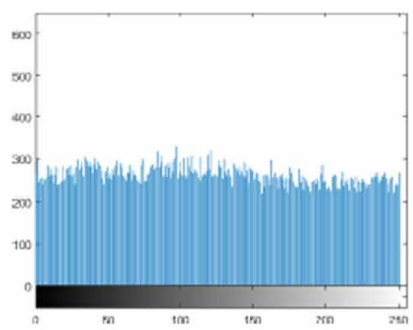

(f)

to the Lagrange interpolation method will also undergo an uncertain change. Similarly, any attack that causes shadow image pixel change will lead to extraction failure. We consider using join error correction codes to improve robustness, which is also our future work.

\subsubsection{Capacity Analyses}

In this scheme, if all the uncertain values are used to hide secret message, the hidden capacity reaches the maximum. According to the formula obtained by quantitative analysis, $E_{p}=$ $(8 \times W \times H \times(k-1)) /(W \times H \times n)=8 \times(k-1) / n B P P$. It's easy to see that the greater the $(k-1) / n$ is, the greater the $E_{p}$ is.

\subsection{Experiment of the Second Scheme}

In this experiment, both the secret image and the secret message are $256 \times 256$ binary images, and we set $(k, k)$ to $(3,3)$, which means the secret image can be recovered only when all the three shadow images are gathered. And since we do not know which shadow image the secret message is embedded in, it is only necessary to get all the shadow images to ensure that the secret message can be extracted.

\subsubsection{Imperceptibility Test}

In the condition of no attack, the experimental results are shown in Figure 5.

The secret image and secret message are given in Figures 5 (a) and (b), and Figure 5 (c) is the encrypted secret message. Figures 5 (d)-(f) display the four shadow images $S C_{1}, S C_{2}$ and $S C_{3}$, which are noisy as well. Figures $5(\mathrm{~g})$ and $(\mathrm{h})$ are the revealed secret image and extracted secret message when collecting $S C_{1}, S C_{2}$ and $S C_{3}$. Image (c) is the result of image (b) encryption. The image (a) is shared into four shadow images (d) (f) using the pixel values in the image (c) as random elements, 
Figure 4. (a) The revealed secret image after additive Gaussian noise; (b)The extracted secret message after additive Gaussian noise; (c) The revealed secret image after additive salt and pepper noise; (d) The extracted secret message after additive salt and pepper noise.

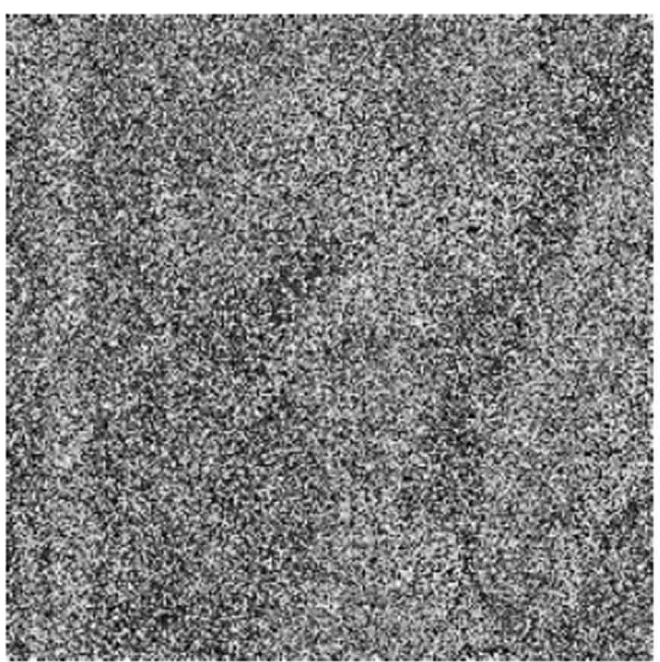

(a)

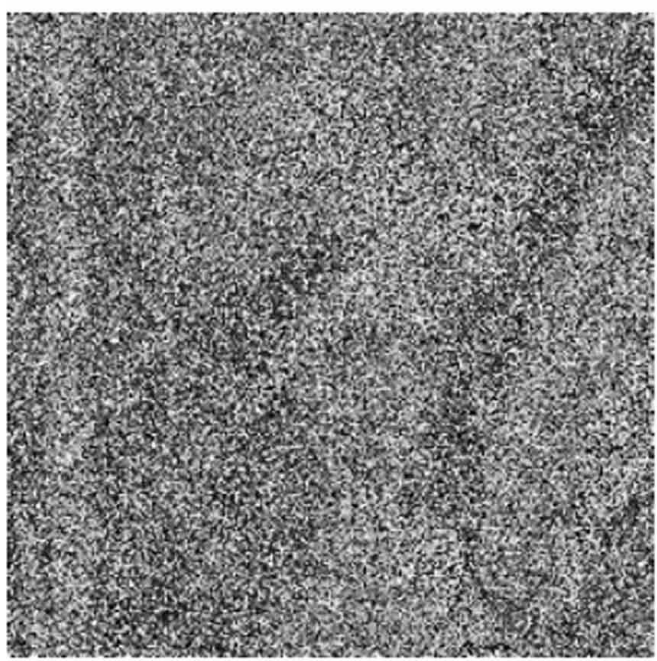

(c)

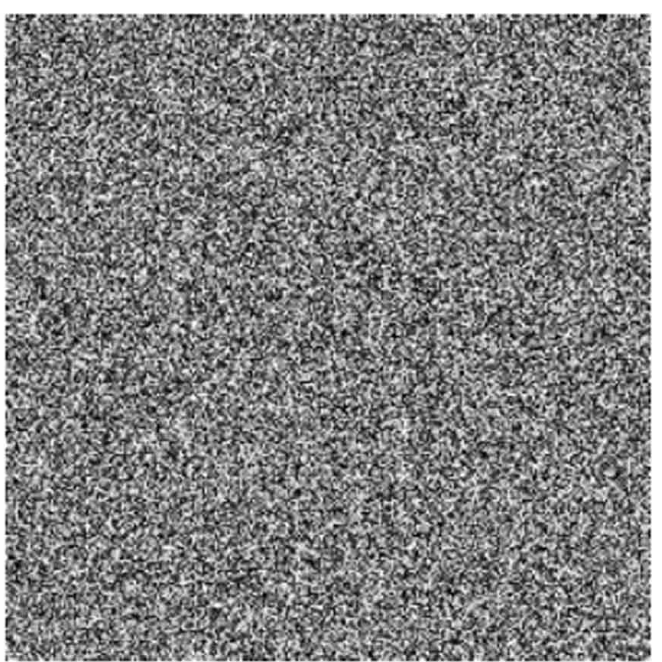

(b)

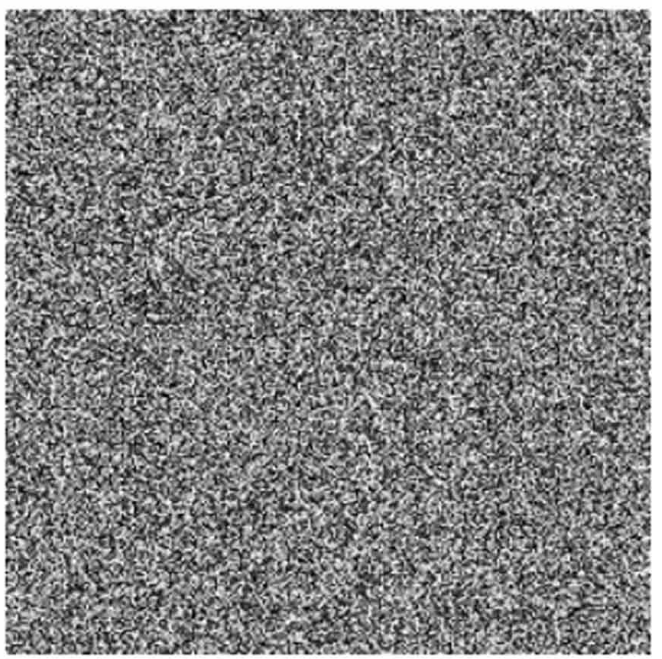

(d)

Table 2. Test results of the proposed scheme

\begin{tabular}{|c|c|c|}
\hline Attacks & BER (\%) & PSNR (db) \\
\hline additive Gaussian noise & 47.33 & 7.4474 \\
\hline additive salt and pepper noise & 50.05 & 7.4654 \\
\hline
\end{tabular}


Figure 5. Experimental results of the proposed scheme based on $(k, k)$ threshold VSS, where $k=3$. (a) The secret image;(b) The secret message;(c)The encrypted secret message;(d)-(e) The first 2 shadow images $S C_{1}, S C_{2}$ to embed the secret messages; (f) The 3th shadow image $S C_{3}$ to compensate the secret image ;(g) Revealing result by $S C_{1}, S C_{2}$ and $S C_{3}$; (h) Extracting result from $S C_{1}$

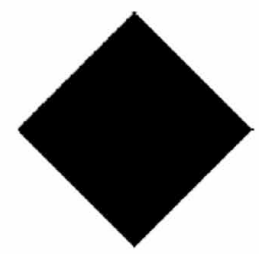

(a)

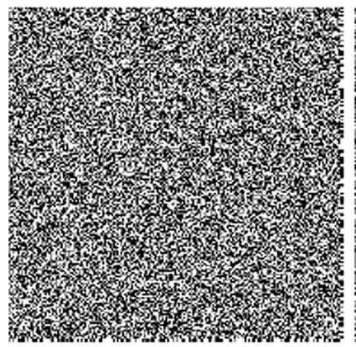

(e)

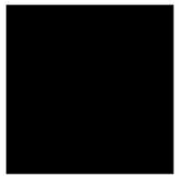

(b)

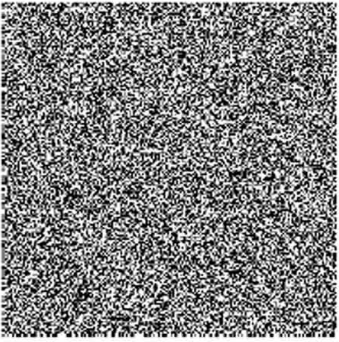

(f)

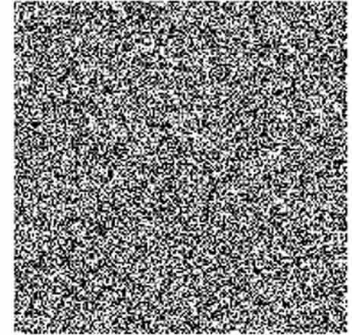

(c)

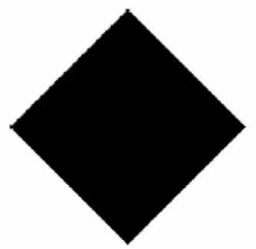

(g)

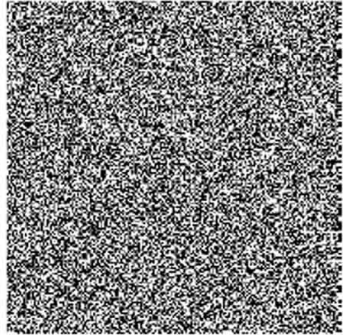

(d)

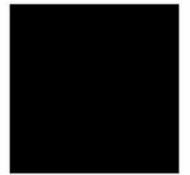

(h)

which is the most important step to realize the transmission of secret image and secret message. When the secret image $(\mathrm{g})$ is recovered by the shadow images $(\mathrm{d})$ and (e), the random element scan be calculated. Therefore, secret message (h) embedded in secret sharing process is extracted.

The histogram of secret image and secret message are shown in Figures 6. (a) and (b), respectively.

And Figures 6. (c) - (e) are the histogram of four shadow images $S C_{1}, S C_{2}$ and $S C_{3}$.

It can be seen from Figure 5 that the encrypted secret message is a noisy image, and the three shadow images are the same. There is nothing related to the secret image and the secret message.

In Figures 6 (a) and (b), the distribution of 1 and 0 is unbalanced, but in shadow images, 1 and 0 are hypo dispersion, which conforms to the characteristics of binary noise image. Thus, according to the results of Figure 5 and Figure 6, we can conclude that the shadow images obtained through this scheme does not reveal the contents of the secret image and secret message, and the scheme has good imperceptibility.

In this experiment, the test results after adding Gaussian noise and salt-pepper noise are shown in Figure 7.

Figures 7 (a) and (b) are the revealed secret image and extracted secret message after additive Gaussian noise. And Figures 7(c) and (d) are the revealed secret image and extracted secret message after additive salt and pepper noise, respectively. Compared to grayscale images, the pixel values of binary images are more likely to change when they suffer from attack, so its ability to resist attacks is worse. Therefore, the robustness of this scheme is also very poor. 
Figure 6. (a) The histogram of secret image; (b) The histogram of secret message; (d) - (e) The histogram of three shadow images $S C_{1}, S C_{2}, S C_{3}$

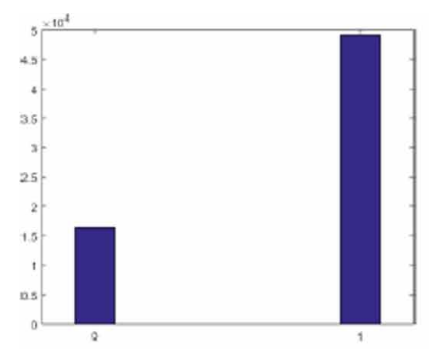

(a)

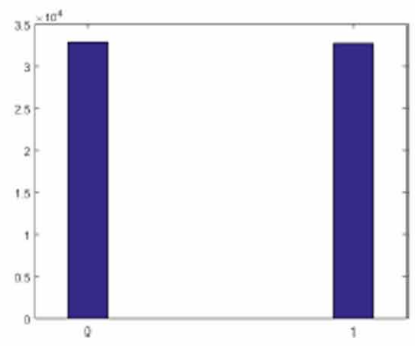

(d)

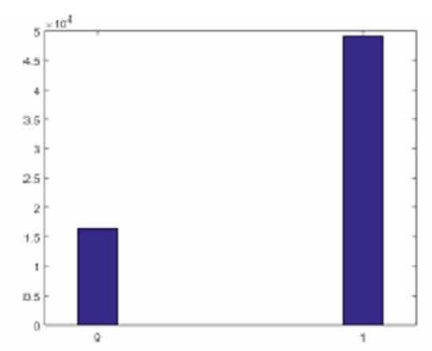

(b)

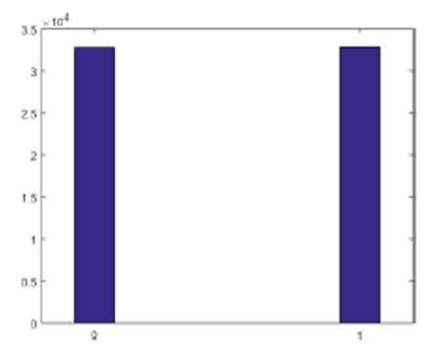

(c)

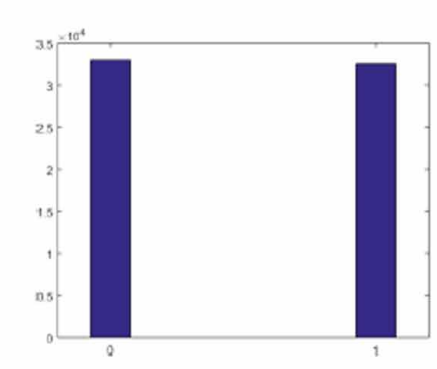

(e)

\subsubsection{Capacity Analyses}

In this scheme, the capacity is related to the number of shadow images which are used to hide messages, and the greater the number is, the greater the capacity is. If there are $v(1 \leq v \leq k-1)$ shadow images that are embedded messages, the capacity is equal to $v \times W \times H$ bits. When $v=k-1$, capacity reaches the maximum and $E_{p}=(k-1) \times W \times H / k \times W \times H=(k-1) / k B P P$. As $k$ increases, $E_{p}$ is getting closer to the capacity of LSB method $(1 B P P)$.

\subsection{Comparison with other Schemes}

Herein, we demonstrate the innovation of our scheme in comparison with $(2,4)$ SIS by experiment. There are two images named Lena and Phone involved in the contrast experiment and the experiment results of $(2,4)$ SIS is shown in Figure 8. and Figure 9.

In Figure 8, image $(a)$ is original image Phone, which is distributed in to four shadow images, image $(b)-(f)$. And we can use image $(b)$ and $(c)$ to restore the original secret image, image $(f)$. Throughout the process, only one secret message, the image named Phone, is transmitted. In Figure 9, image $(a)$ is original image Lena, which is distributed into four shadow images, image $(b)-(f)$. And we can use image $(b)$ and $(c)$ to restore the original secret image, image $(f)$. Throughout the process, only one secret message, the image named Lena, is transmitted. But from Figure 2 and Figure 5 , we can see that there are two secret messages are transmitted in our schemes. This is the innovation between our proposed scheme and previous works such as SIS and VSS.

Compared with traditional steganography methods, the advantages of our model are summarized as follows: 
Figure 7. (a) The revealed secret image after additive Gaussian noise; (b) The extracted secret message after additive Gaussian noise; (c) The revealed secret image after additive salt and pepper noise; (d) The extracted secret message after additive salt and pepper noise

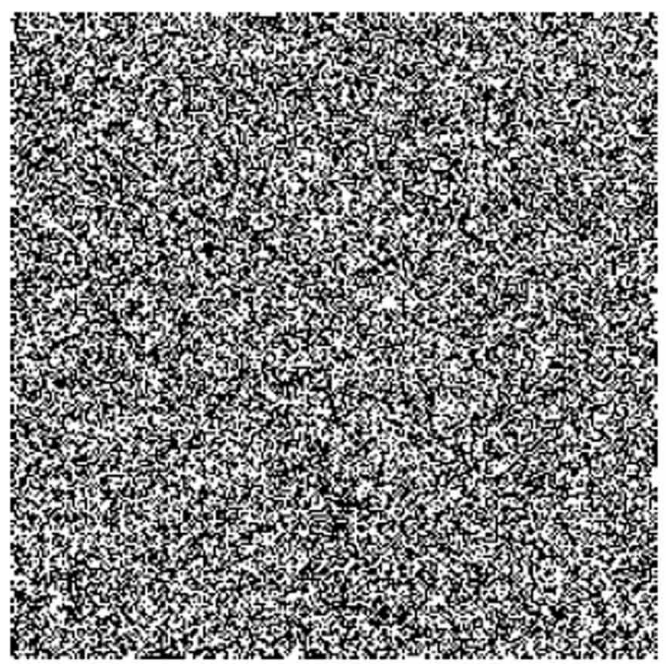

(a)

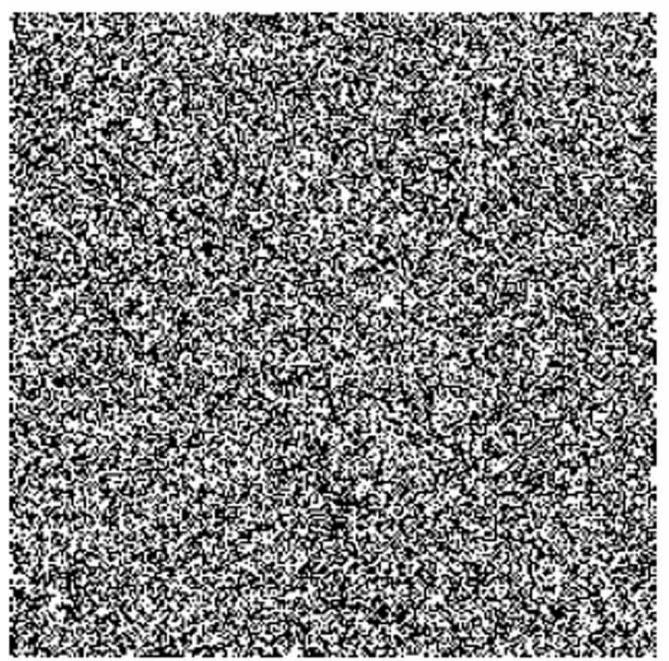

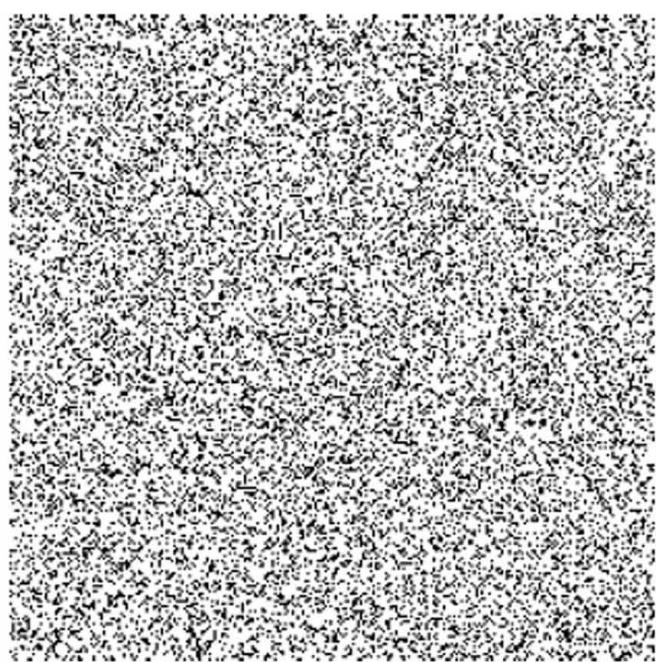

(b)

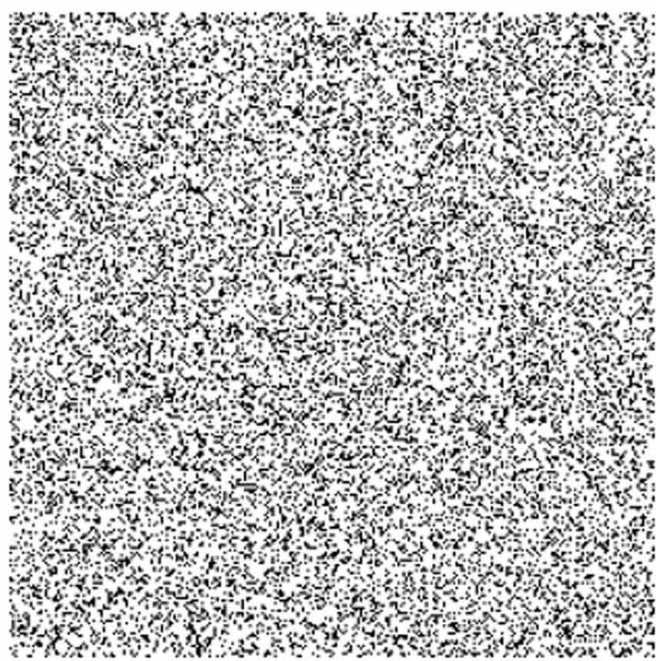

(d)

Taking the first scheme as an example, since it does not require any original carrier, this scheme has better imperceptibility than other scheme. But the robustness is not even as good as LSB method. Compared with $E_{p}=1 B P P$ of traditional LSB method, the capacity of this scheme is much larger. And compared with the method proposed by Zhang in (Zhang, 2017) whose capacity is one bit per behavior, the capacity of our scheme is also more advantageous. 
Figure 8. (a) The secret image Phone; (b) - (e) Four shadow images $S C_{1}, S C_{2}, S C_{3}, S C_{4}$ of image Phone; (f) Revealing result by $S C_{1}$ and $S C_{2}$

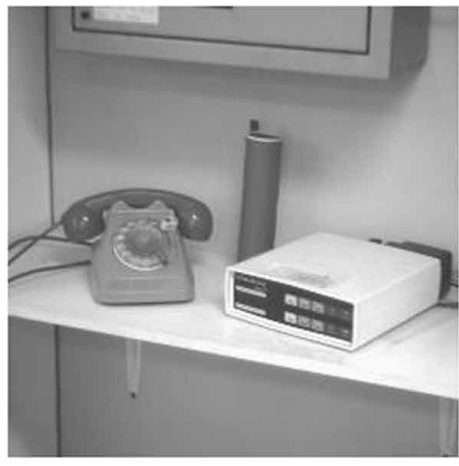

(a)

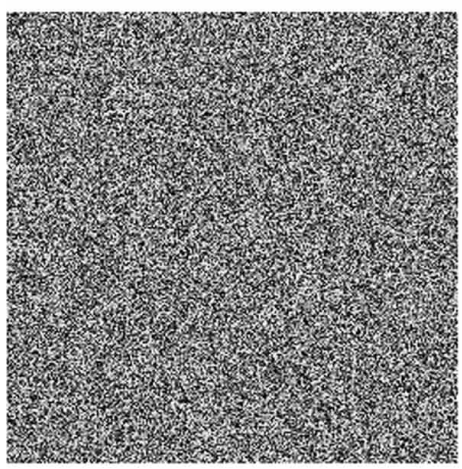

(d)

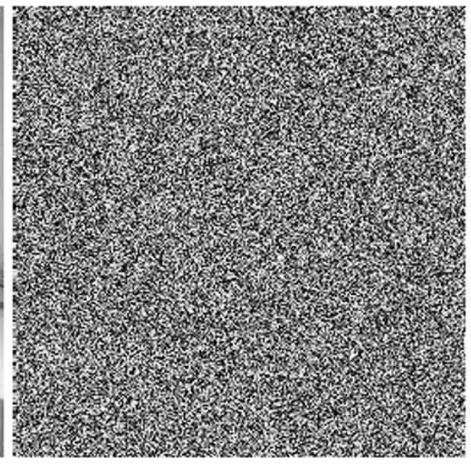

(b)

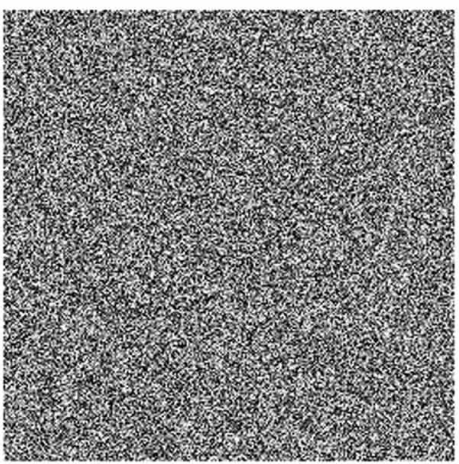

(e)

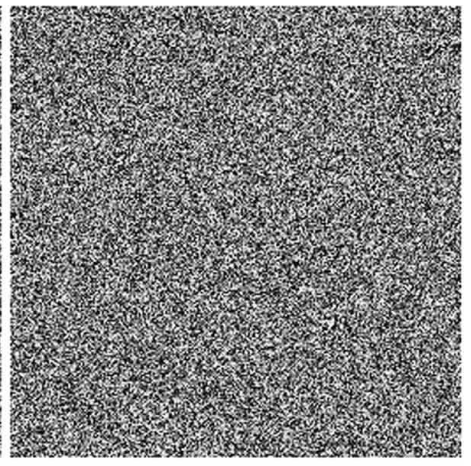

(c)

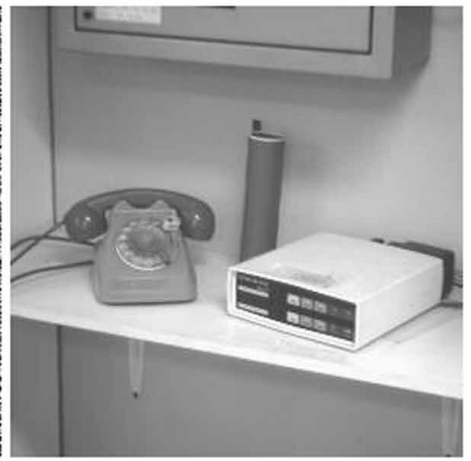

(f)

\section{CONCLUSION}

This paper presents a novel behavior steganography model based on secret sharing, which is applicable to a variety of secret sharing algorithms and implements two channel secret information transmission, including information hiding and secret sharing. Based on two secret image sharing algorithms- $(k, n)$ threshold SIS and $(k, k)$ threshold VSS, two specific behavior steganography schemes are proposed. Their common feature is that the original carrier is not needed, and secret messages are hidden by selecting random values. Experiments and comparisons with other scheme show that the proposed schemes have a larger capacity than traditional steganography methods. Moreover, two schemes not only have good imperceptibility, but also don't affect the recovery of secret images and the extraction of secret messages. The drawback is that they have poor robustness for resisting common attacks. Therefore, We will study how to improve the robustness of our schemes and utilize other secret sharing algorithms to achieve behavior steganography as the proposed model in the future. 
Figure 9. (a)The secret image Lena;(b)-(e)Four shadow images $S C_{1}, S C_{2}, S C_{3}, S C_{4}$ of image Lena; (f) Revealing result by $S C_{1}$ and $S C_{2}$

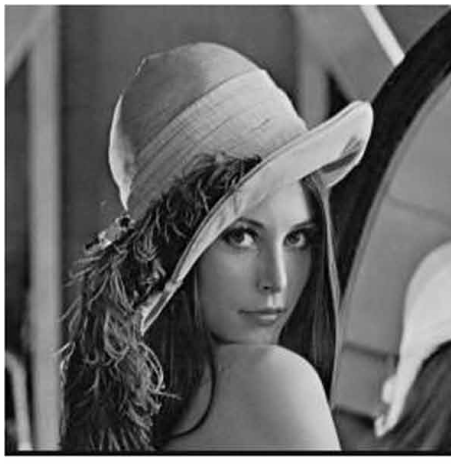

(a)

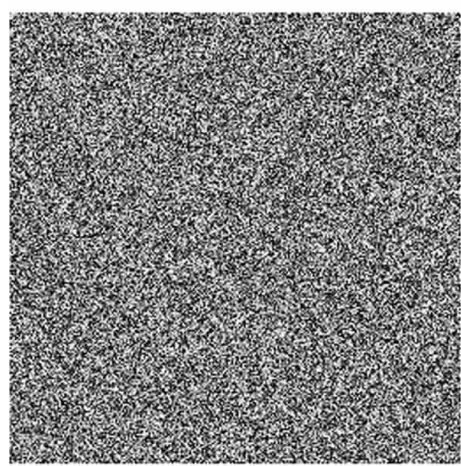

(d)

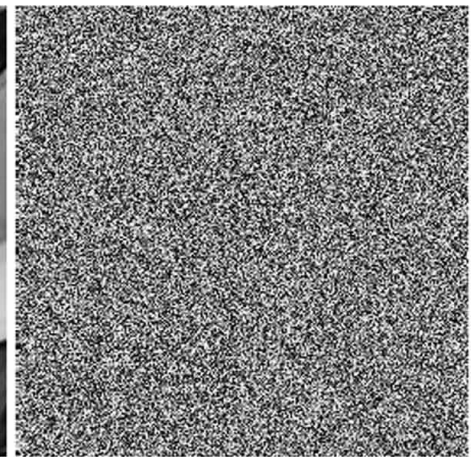

(b)

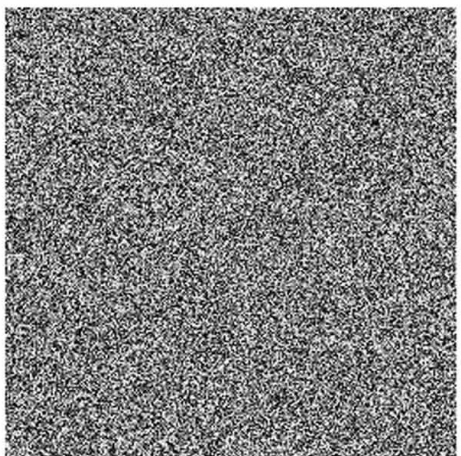

(e)

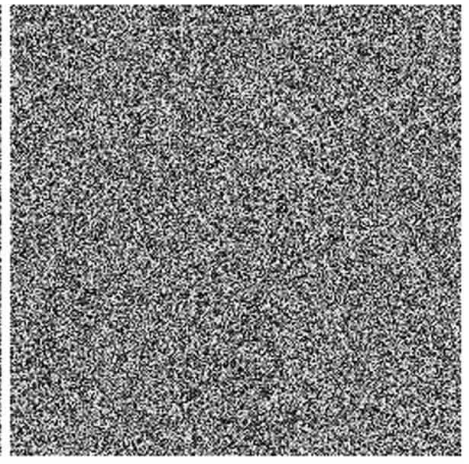

(c)

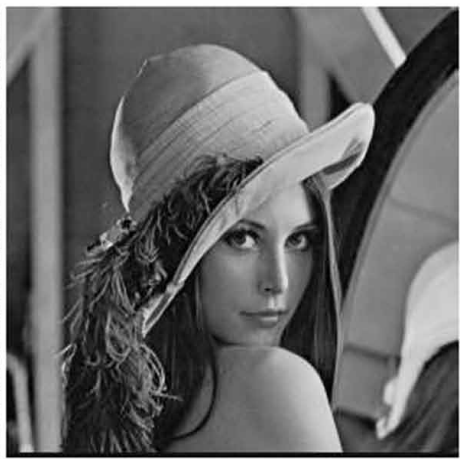

(f)

\section{ACKNOWLEDGMENT}

The authors would like to thank the anonymous editor and reviewers for their valuable comments. This work is supported by the National Natural Science Foundation of China (Grant Number: 61602491) and Key Program of National University of Defense Technology. 


\section{REFERENCES}

Banik, B. G., \& Bandyopadhyay, S. K. (2016). Secret sharing using 3 level dwt method of image steganography based on lorenz chaotic encryption and visual cryptography. In International Conference on Computational Intelligence and Communication Networks.

Cao, R.B. (2011). Binary image hiding algorithm based on multi-secret sharing and dct. Computer Science, $38,270-284$.

Divya, S. S., \& Reddy, M. R. M. (2012). Hiding text in audio using multiple LSB steganography and provide security using cryptography. International journal of scientific \& technology research, 1(6), 68-70.

Jagadeesh, N., Nandakumar, A., Harmya, P., \& Anju, S. S. (2011). Secret image sharing using steganography with different cover images. Communications in Computer and Information Science, 191, 490-497. doi:10.1007/9783-642-22714-1_50

Johnson, N. F., Duric, Z., \& Jajodia, S. (2012). Information Hiding: Steganography and Watermarking-Attacks and Countermeasures. Springer.

Kafri, O., \& Keren, E. (1987). Encryption of pictures and shapes by random grids. Optics Letters, 12(6), 377-379. doi:10.1364/OL.12.000377 PMID:19741737

Khosravi, M. J., \& Ghandali, S. (2011). A secure joint wavelet based steganography and secret sharing method. In International Conference on Information Assurance and Security (pp. 222- 227). doi:10.1109/ ISIAS.2011.6122823

Kong, J., Zhang, Y., Meng, X., Zheng, Y., \& Lu, Y. (2007). A Scalable Secret Image Sharing Method Based on Discrete Wavelet Transform. Springer Berlin Heidelberg. doi:10.1007/978-3-540-74769-7_78

Li, L., El-Latif, A. A. A., Yan, X., Wang, S., \& Niu, X. (2012). A lossless secret image sharing scheme based on steganography. In Second International Conference on Instrumentation, Measurement, Computer, Communication and Control (pp. 1247-1250). doi:10.1109/IMCCC.2012.293

Li, P., Ma, P., Su, X., \& Liu, F. (2012). Multi-threshold image secret sharing scheme. Acta Electronica Sinica, $40,518-524$.

Li, P., Yang, C.-N., Wu, C.-C., Kong, Q., \& Ma, Y. (2013). Essential secret image sharing scheme with different importance of shadows. Journal of Visual Communication and Image Representation, 24(7), 1106-1114. doi:10.1016/j.jvcir.2013.07.005

Lin, S.-J., \& Lin, J.-C. (2007). Vcpss: A two-in-one two-decoding-options image sharing method combining visual cryptography (vc) and polynomial-style sharing (pss) approaches. Pattern Recognition, 40(12), 3652-3666. doi:10.1016/j.patcog.2007.04.001

Naor, M., \& Shamir, A. (1995). Visual cryptography. In Advances in Cryptology EUROCRYPT'94 (pp. 1-12). Springer Perugia, Italy: Springer. doi:10.1007/BFb0053419

Shamir, A. (1979). How to share a secret. Communications of the ACM, 22(11), 612-613. doi:10.1145/359168.359176

Thien, C.-C., \& Lin, J.-C. (2002). Secret image sharing. Computers \& Graphics, 26(5), 765-770. doi:10.1016/ S0097-8493(02)00131-0

Wang, Z., Arce, G. R., \& Di Crescenzo, G. (2009). Halftone visual cryptography via error diffusion. IEEE Transactions on Information Forensics and Security, 4(3), 383-396. doi:10.1109/TIFS.2009.2024721

Yan, X., Wang, S., \& Niu, X. (2014). Threshold construction from specific cases in visual cryptography without the pixel expansion. Signal Processing, 105, 389-398. doi:10.1016/j.sigpro.2014.06.011

Yang, C.-N., \& Ciou, C.-B. (2010). Image secret sharing method with two-decoding-options: Lossless recovery and previewing capability. Image and Vision Computing, 28(12), 1600-1610. doi:10.1016/j.imavis.2010.04.003

Zhang, X. (2017). Behavior Steganography in Social Network. Springer.

Zhang, X. P., Qian, Z. X., \& Li, S. (2016). Prospect of digital steganography research. 
Zieliska, E., Mazurczyk, W., \& Szczypiorski, K. (2014). Trends in steganography. Communications of the ACM, 57(3), 86-95. doi:10.1145/2566590.2566610

Hanlin Liu was born in China, in Aug 1993, received the B.Sc. degree with honor rank in Computer Application, China in 2015. He is now a postgraduate student at Hefei electronic engineering institute, Hefei, P. R. China. His areas of interests are multimedia security, information hiding.

Jingju Liu was born in China, in 1974, received the B.Sc. degree with honor rank in Computer Application, China in 1996 and M.Sc. degree in Computer Application in 2001 from Hefei electronic engineering institute. She now is a professor at National University of Defense Technology, Hefei, P. R. China. Her areas of interests are network security.

Xuehu Yan was born in China, in Feb 1984, received the B.Sc. degree with honor rank in Science in Information \&; Calculate Science, China in 2006, M.Sc. degree in Computational Mathematics in 2008, and doctoral degree in Computer Science and Technology in 2015 from Harbin Institute of Technology. He now is a lecture at National University of Defense Technology, Hefei, P. R. China. His areas of interests are cryptography, multimedia security, secret image sharing and biometrics.

Lintao Liu was born in China, in Dec 1989, received the B.Sc. degree with honor rank in Computer Application, China in 2012, M.Sc. degree in Information Security in 2015 from Hefei electronic engineering institute. He now is a PhD candidate at National University of Defense Technology, Hefei, P. R. China. His areas of interests are cryptography, multimedia security, secret image sharing and biometrics.

Wanmeng Ding was born in China, in Feb 1994, received the B.Sc. degree with honor rank in Computer Application, China in 2015. She is now a postgraduate student at National University of Defense Technology, Hefei, P. R. China. Her areas of interests are multimedia security, secret image sharing and computer application.

Yue Jiang was born in China, in Feb 1989, received the B.Sc. degree with honor rank in Information Engineering, China in 2010 from Linyi University and M. Sc. degree in Technology of Computer Application in 2013 from Beijing Technology and Business University. She now is an assistant at National University of Defense Technology, Hefei, P. R. China. Her areas of interests are visual cryptography and multimedia security. 